This item was submitted to Loughborough's Research Repository by the author.

Items in Figshare are protected by copyright, with all rights reserved, unless otherwise indicated.

\title{
Designing PI controllers for TCP/IPv6 networks supporting differentiated QoS requirements
}

PLEASE CITE THE PUBLISHED VERSION

\section{PUBLISHER}

(C) IEEE

\section{VERSION}

VoR (Version of Record)

\section{LICENCE}

CC BY-NC-ND 4.0

\section{REPOSITORY RECORD}

Li, Yunqiu, and Shuang-Hua Yang. 2019. "Designing PI Controllers for Tcp/ipv6 Networks Supporting Differentiated Qos Requirements". figshare. https://hdl.handle.net/2134/5117. 
This item was submitted to Loughborough's Institutional Repository (https://dspace.lboro.ac.uk/) by the author and is made available under the following Creative Commons Licence conditions.

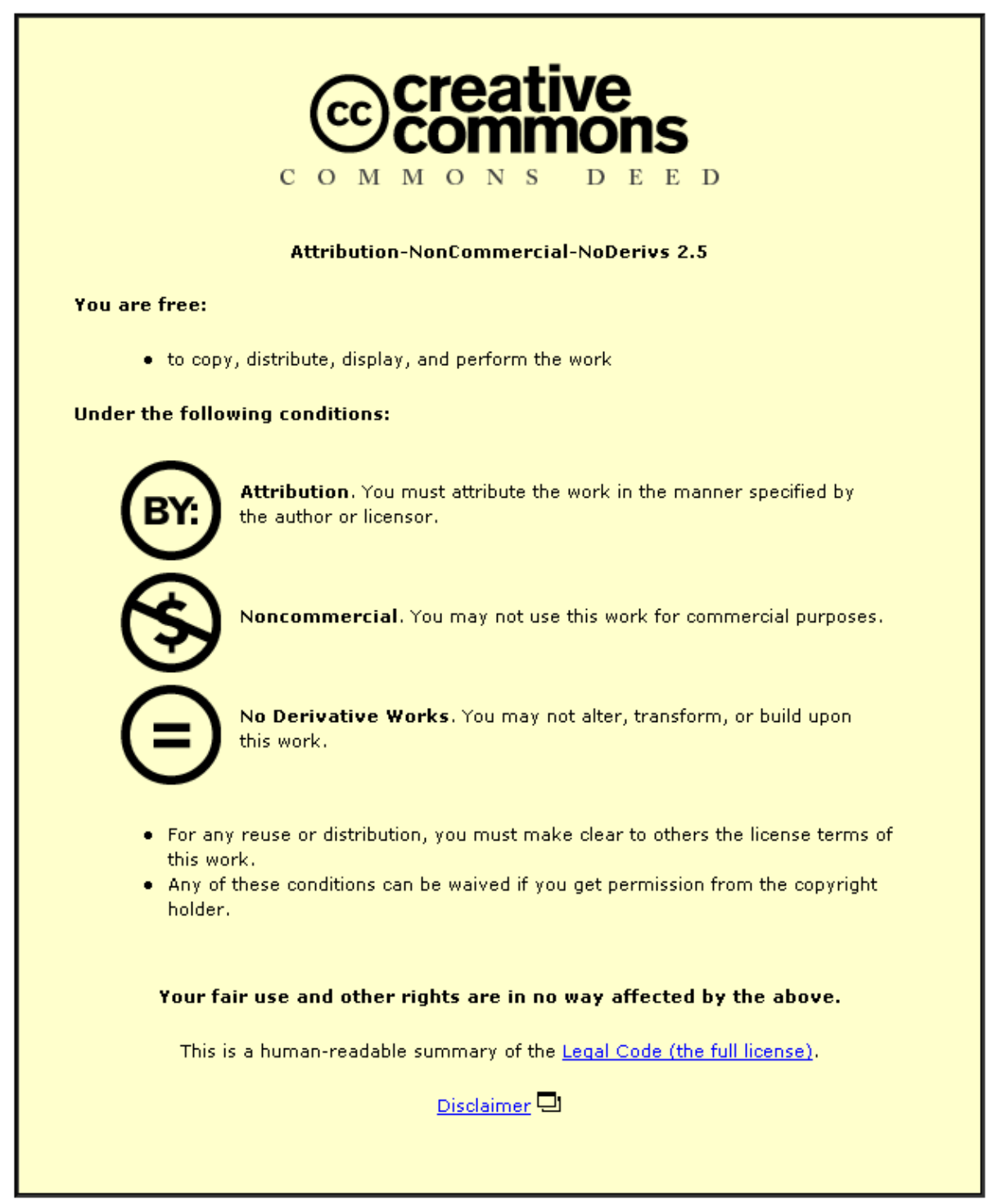

For the full text of this licence, please go to: http://creativecommons.org/licenses/by-nc-nd/2.5/ 


\title{
Designing PI Controllers for TCP/IPv6 Networks Supporting Differentiated QoS Requirements
}

\author{
Y. LI, Student Member of IEEE, Prof. S.H. Yang, Senior Member, IEEE
}

\begin{abstract}
This paper presents a set of controllers for TCP/IPv6 networks. These controllers are developed from the traditional Proportional Integral Controller, and can support differentiated Quality of Service Requirement of the end users. Those requirements are labeled in the IPv6 header of the packets, checked by the controllers upon their arrival to the routers, so as to provide guidance to further dropping actions when congestions occur. Simulation results demonstrate the controllers' ability of guaranteeing certain levels of Quality of Service to the end users according to their demands.
\end{abstract}

\section{INTRODUCTION}

$\mathrm{I}_{\mathrm{h}}^{\mathrm{N}}$ NTERNET congestion has become an obstacle to providing high-leveled Quality of Service (QoS) to certain end users, as a result of the explosive growth in Internet data transmission. Research on Internet congestion control comprises two main parts: Transmission control protocol (TCP) and Active Queue Management (AQM) [1]. Many AQM algorithms have been proposed over the last decade. Random Early Detection (RED) [2] is an AQM algorithm widely implemented in major commercial routers. The implementation of RED could avoid TCP global synchronization and provide better QoS to the end users. Although intensive research has been done to investigate RED [3], there are some questions over RED implementations [4], and some limitations of RED have been identified. [5].

The IPv4 header has been acting as an assisting component to several AQM algorithms, such as Weighted RED (WRED) [6] and RED with In/Out bit (RIO) [7], to introduce some QoS consideration to the transmission. However, the growth of the Internet also pressurizes the Internet Engineering Task Force (IETF) to upgrade Internet Protocol IPv6 to satisfy the large demands for IP addresses. IPv6 completely revamps IPv4, addressing performance, scalability, security, etc. The 20 bits flow label field, which is a new field added in IPv6 header, is used to facilitate identification of data requiring special handling, such as those arising from real-time applications, etc.

Having the availability of the new flow label field, Active Queue Management algorithms with Priority Checking

Manuscript received October 13, 2008. This work was supported by Computer Science Department, Loughborough University.

Y. Li, is PhD student with Computer Science Department, Loughborough University, Loughborough, Leicestershire, UK, LE11 3TU (e-mail: Y.LI3@1boro.ac.uk, phone: +44(0)1509635648)

S.H. Yang, is Professor with Computer Science Department, Loughborough University, Loughborough, Leicestershire, UK, LE11 3TU

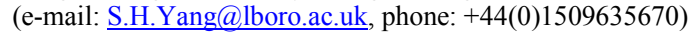

Mechanism (PC-AQM), such as PC-RED [8] are developed to ensure that the end user with high priority to get the level of QoS they expected. This can be achieved by implementing a Priority Checking function in AQM, in which the flow label field is used to label the packets of different dataflow with their priority and the current level of services they have experienced, so they can request from the network router with PC-AQM distinct QoS. Thus, real-time system users or people who would like to pay more for better services can obtain a guaranteed high level of QoS.

However, the slower response and slightly larger oscillation caused by the priority checking, the dropping probability calculation function, and the parameter tuning problem inherited from traditional RED are common shortcomings of RED-based PC-AQM algorithms. In this paper, we combine the priority checker and the packet labeler with a traditional PI controller for AQM to improve system performance.

The rest of the paper is organized as follows. Section II discussed related works on AQM. Section III and IV focus on PI controller with Priority Checking function (PC-PI) and Double Status PC-PI controller (DSPCPI), which can reduce the overshoot of the queue size while the network work load varies, respectively. To make up the extra time delay caused by the Priority Checking Mechanism, a PC-PI controller with Prediction Mechanism (KPCPI) is presented in Section V. Simulation results obtained by using Network Simulator 2 (NS2) and SIMULINK are presented in Section VI. The simulations illustrate the results of guaranteeing some form of QoS to particular end user with special QoS Requirement. Finally, the conclusions are given in Section VII.

\section{RELATED WORKS}

\section{A. RED and PC-RED}

RED algorithm detects potential congestion by calculating the average queue length. It can then notify connections to avoid congestion either by dropping packets arriving at the gateway or by setting one bit in the packet headers to 1 or 0 . RED algorithm consists of two main parts, calculating the average queue length and calculating the probability of dropping an incoming packet. Because the packet bursts are part of the characteristics of networking, RED calculates the average queue length using a type of a low-pass filter with an exponential weighted moving average. So, the average queue length equals:

$$
q_{\text {ave }}=(1-w) \times q_{\text {ave }}+q_{\text {cur }} \times w
$$


$q_{\text {ave }}$ is the average queue size, updated every packet arrival. $q_{\text {cur }}$ is the current queue size, and $w$ is the queue weight, usually set as 0.002 .

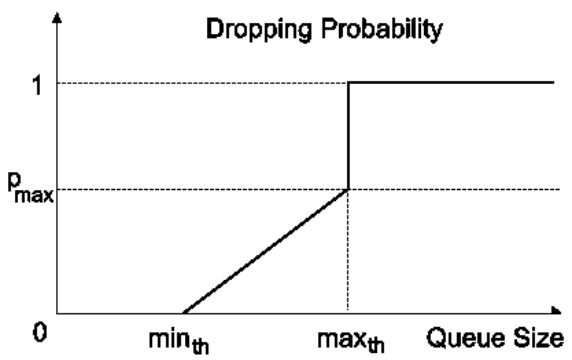

Fig. 1. Dropping Probability in RED

There are two thresholds needed while calculating the dropping probability in RED. The $\min _{\text {th }}$ denotes the minimum threshold while the $\max _{t h}$ denotes the maximum threshold. Figure 1 shows how the probability changes while the average queue length varies.

The QoS Requirement can be labeled in the packet header in IPv6 network. In the PC-RED router, an extra database is set up referred to as the Priority List, which contains the QoS information carried by the packet header along with the data generated by the router itself. Compare to traditional AQM algorithms, the structure of PC-RED has the extra functionality of checking and labeling the IPv6 headers with QoS related information based on the Priority List, which is taken into account by the discard tester during congestion.

PC-RED updates this list when packets reach the interface. A count down timer is used to trigger the Priority List Cleaner function. If one entry of the list has not been updated during the last count down timer interval, PC-RED would consider this dataflow dead and delete the entry. The average queue length is calculated upon every packet arrival. The probability that an incoming packet would be dropped depends on the combined value of two parts, the dropping probability generated from the average queue length using the traditional RED probability calculation function, and the dropping suggestion held in its IPv6 header labeled by PC-RED referencing the Priority List.

PC-RED can guarantee demanded QoS level to the end users. However, the parameter tuning problem inherited from traditional RED together with the slower response and slightly larger oscillation caused by the priority checking function are its shortcomings.

\section{B. $P I-A Q M$}

Another AQM algorithm developed from the classical control system techniques, the Proportional Integral controller is said to be a natural choice due to its robustness and its ability to eliminate the steady-state error. Although it PI is difficult to implement, compared to RED, PI still has some advantages; it does not need very complicated parameterization, and it provides the ability to efficiently control queue size under heavy work loads. PI attempts to maintain an explicit target queue length $q_{r e f}$. It samples instantaneous queue length at fixed intervals and computes a dropping probability $p_{p i}$ for the incoming packet.

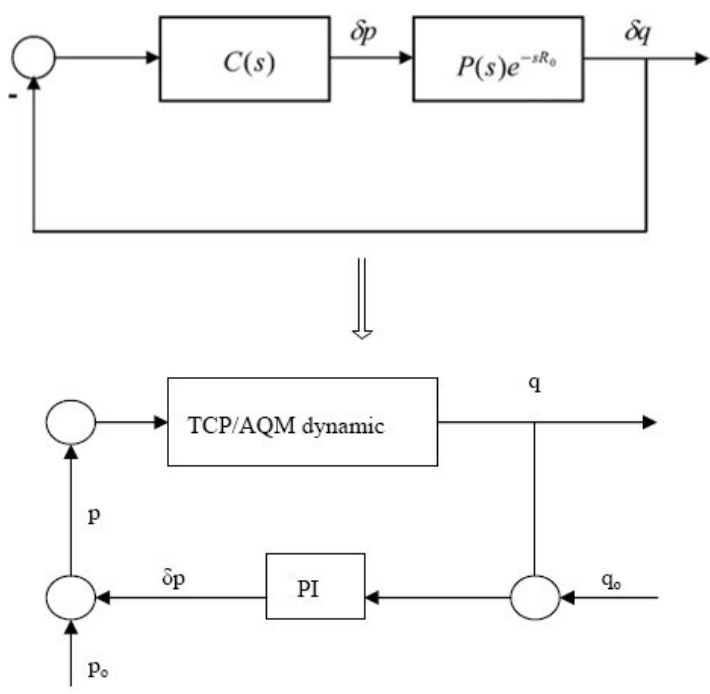

Fig. 2. PI AQM Block Diagram [9]

A simplified version of a dynamic TCP model using fluid-flow and stochastic differential equation analysis is used and linearized in the PI controller. It ignores both the Slow Start phase and the timeout mechanism of TCP. $C(s)$ is the transfer function of traditional AQM controller, such as RED. $P(s)$ is the transfer function of TCP windows and queue size. $R_{0}$ is the network delay. Using this TCP/AQM dynamics, the PI controller is expected not only to improve the responsiveness of the TCP/AQM dynamics but also as to stabilize the router queue length around the desired value. The latter can be achieved by means of integral control, while the former can be achieved by means of proportional control using instantaneous queue length instead of using the average queue length. The procedure of implementation in the AQM Enabled router is shown in Figure 3.

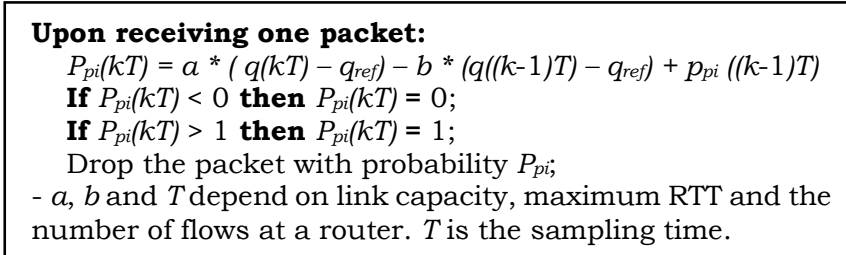

Fig. 3. PI AQM Algorithms

\section{PRIORITY CHECKING PI-AQM (PCPI)}

In TCP/IPv6 networks, to dataflows with different priorities, the Proportional Integral controller can work with Priority Checking function to divide the dropping probability into different ranks.

QoS Requirement is labeled in the IPv6 header. One extra bit in the IPv6 header, which is referred to as QoSA (Quality of Service Alert), is also used to indicate whether the dataflow has been treated worse than was expected. In PC-PI, the priority list contains the current status of every data flow passing through the router, as shown in Table I. The tagger will then mark the flow label field with regards to the information that the list carries. 
TABLE I

PRIORITY LIST ENTRY

\begin{tabular}{|c|c|c|c|c|c|}
\hline 1. & \multirow{2}{*}{$\begin{array}{c}\text { 2. QoS } \\
\text { Flow }\end{array}$} & \multicolumn{2}{|c|}{3 . Average Dropping Rate } & 4. \\
\cline { 3 - 4 } & Requirement & $\begin{array}{c}\text { Drop } \\
\text { Counter }\end{array}$ & $\begin{array}{c}\text { Arrival } \\
\text { Counter }\end{array}$ & $\begin{array}{c}\text { Weight } \\
\text { Factor }\end{array}$ & Status \\
\hline
\end{tabular}

1. Flow Id $\left(f_{i d}\right)$ : Unique identification of every dataflow.

2. QoS Requirement $\left(q_{r}\right)$ : Current QoS requirement defined by the end user.

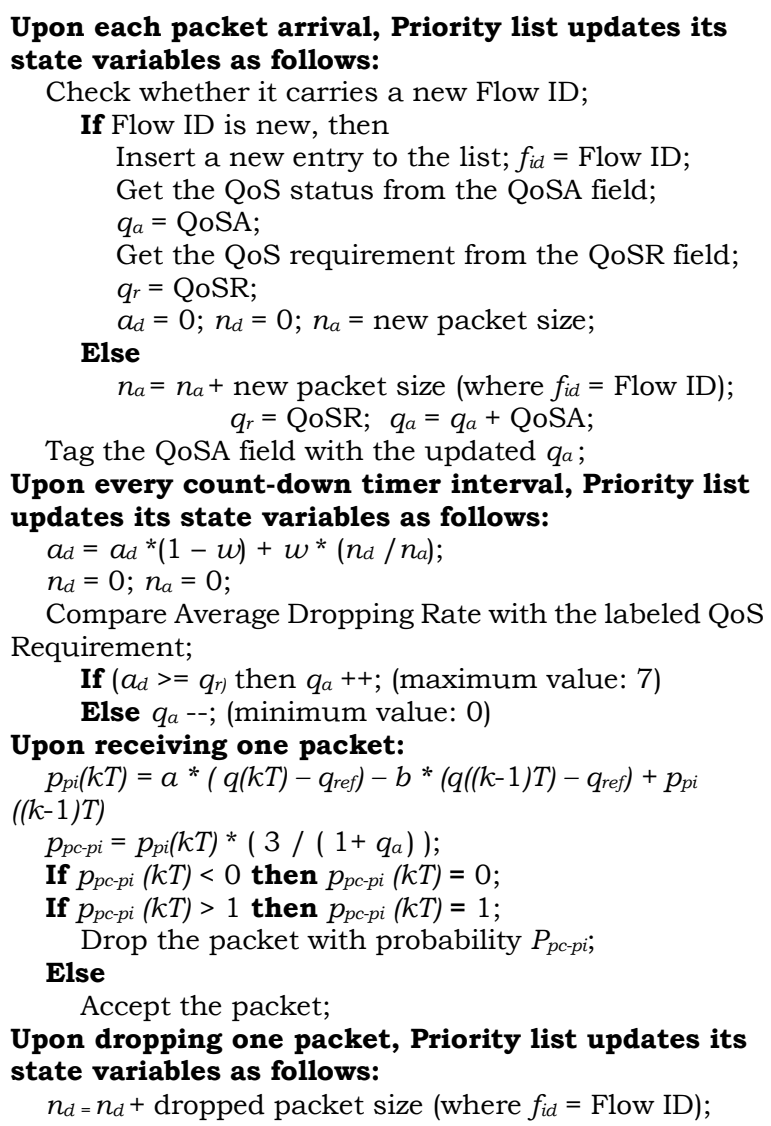

Fig. 4. PC-PI Algorithms

3. Average Dropping Rate $\left(a_{d}\right)$ : Weighted Moving Average of the ratio of the amount of dropped packets to the amount of arrived packet of the same flow during every time interval.

3.1 Dropped Counter $\left(n_{d}\right)$ : The amount of dropped packets.

3.2 Arrived Counter $\left(n_{a}\right)$ : The amount of arrived packets.

3.3 Weight Factor $(w)$ : Weight factor that is used to calculate the Average Dropping Rate.

4. Current Treatment $\left(q_{a}\right)$ : Current QoS status that the data flow is being treated in.

The tagging algorithms of PC-PI are rather similar to the PC-RED algorithms of the previous section, apart from the buffer management and packet dropping function. The difference is that the former does not have two queue size threshold $\min _{t h}$ and $\max _{t h}$. The $q_{\text {ref }}$ is considered as the set-point in the traditional PI controller. The PC-PI controller first generates a generic dropping probability $\mathrm{P}_{\mathrm{pi}}$ for all incoming packet based on the difference between the queue size and the $q_{r e f} . p_{p i}$ is then used in conjunction with the QoS Alert carried in the IPv6 header to generate the dropping probability $p_{p c-p i}$ for each chosen packet.

\section{DOUBLE STATUS PC-PI (DSPCPI)}

PC-PI works satisfactory when the network load level is stable. However, when there are many dataflows dropping out of the network or transmitted into the network, large oscillations of the queue size occur. Hence, the overall throughput would be reduced, which leads to a larger number of dataflows carrying QoS Alert warnings. To improve the QoS under unstable work load, Double Status Priority Checking PI controller (DSPCPI) is proposed.

$$
\begin{aligned}
& \text { Upon each count down timer interval: } \\
& q_{\text {ave }}=(1-w) \times q_{\text {ave }}+q_{\text {cur }} \times w \\
& \text { if }(1+0.25) \times q_{r e f}<q u e u e \text { buffer } \\
& \quad q_{s w}=q_{r e f}+0.25 \times q_{r e f} \\
& \text { else } \\
& \quad q_{s w}=q_{r e f}+\frac{q u e u e \text { buffer }-q_{r e f}}{2} \\
& q_{r e f 2}=q_{r e f}-0.08 \times q_{\text {ref }}
\end{aligned}
$$

If average queue size is under the switch queue size qsw:

Consider the status as Normal;

Calculate the dropping probability as PC-PI does; Else

Consider the status to be Critical;

$$
k_{1}=\frac{k_{2} \times\left(q_{c u r}-q_{r e f 2}\right)}{1+p_{c r i}}, p_{c r i}=k_{1} \times\left(q_{\text {ave }}-q_{r e f 2}\right)
$$

$q_{s w}:$ the switch between two control statuses;

$q_{\text {ref }}$ : the target queue size under Normal control status;

$q_{\text {ref } 2}:$ the target queue size under Critical control status;

$k_{1}$ : dropping probability updating variable;

$k_{2}$ : dropping probability updating parameter, $5 e-4$.

$p_{p i}$ : dropping probability used under Normal control status;

$p_{c r i}$ : dropping probability used under Critical control status;

Fig. 5. DSPCPI Algorithms

The algorithm of DSPCPI is shown in Figure 5. A PI controller is used when the workload is stable, so as to restrict the queue size to a certain range. When there is large fluctuation in the workload, we use a different control status to rapidly reduce the system response time.

The main idea of using two control statuses is to fasten the response time while the queue length gets out of reasonable range due to the rapid change of the network workload. When there is a large amount of flows leaving the network, since it will not cause any congestion, the DSPCPI does not response. Hence the switch between two control statuses can only be triggered when the average queue size exceed a predetermined value. We use average queue size instead of current queue size because as the work load of the network changes, the queue size fluctuates, and the current queue size can be rather unstable. Bringing a low-pass filter into the system can reduce the oscillation, hence gives the system enough time to response to the new dropping probability.

$k_{l}$ is the dropping probability updating variable. It is 
proportional to the difference value between the current queue size and the target queue size. Since we do not want DSPCPI to over-drop packets, when taking the previous dropping probability into account, we suggest $k_{l}$ should be reduced by a certain rate related to $p_{c r i}$.

\section{PC-PI WiTH KALMAN PREDICTOR (KPCPI)}

When adding the extra Priority Checking and Packet Header Labeling mechanism to the router, the delay of the network increases. We can use a prediction mechanism to make up on this.

A dynamic model of TCP behaviour was developed using fluid-flow and stochastic differential equation analysis in [2]. Based on that, a simplified TCP-queue dynamic model is presented.

$$
\left\{\begin{array}{l}
\dot{W}(t)=\frac{1}{R(t)}-\frac{W(t) \times W(t-R(t))}{2 R(t-R(t))} \times p(t-R(t)) \\
\dot{q}(t)=\frac{W(t)}{R(t)} \times N(t)-C
\end{array}\right.
$$

Where $\dot{x}$ denotes the time-derivative of $x$, written as

$\dot{x}(t)=\frac{\partial}{\partial t} x(t)$, and

$W=$ expected TCP window size in packets;

$q=$ expected queue length in packets;

$R=$ round-trip time $=\frac{q}{C}+T_{p}($ secs $) ;$

$C=$ link capacity in packets per second;

$T_{p}=$ propagation delay in seconds;

$N=$ load factor, indicating the number of TCP sessions in total;

$p=$ the probability of packet dropping;

When introducing a Priority Checking Mechanism into the system. Dataflows with different QoSR field and QoSA field will be assigned different dropping probabilities. Thus, based on (1), the block diagram of the system can be transform into the following format.

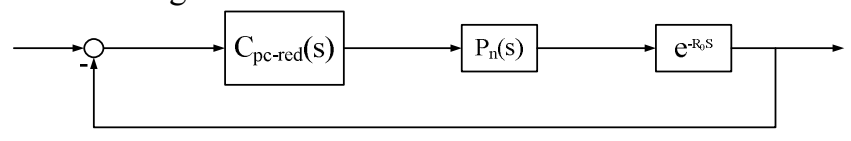

Where

Fig. 6. PC-RED Block Diagram

$K_{n}=1-K_{p}$

$K_{p} \in\left[0, \frac{r_{p}}{q}\right]$ when all High-Priority flows are holding 1 as QoS Alert.

$r_{p}$ is the ratio of High-Priority flow to the overall load level $\mathrm{N}$.

$$
P_{n}(s)=\frac{\frac{R_{0} C^{2}}{2 N_{n}^{2}}}{s+\frac{2 N_{n}}{R_{0}^{2} C}} \cdot \frac{\frac{N_{n}}{R_{0}}}{s+\frac{1}{R_{0}}}=\frac{\frac{C^{2}}{2 N_{n}}}{\left(s+\frac{2 N_{n}}{R_{0}^{2} C}\right)\left(s+\frac{1}{R_{0}}\right)}
$$

$$
\begin{gathered}
C_{p c \text { red }}(s)=\frac{\frac{1-K_{p}}{1-r_{p}}\left(s+\frac{2 r_{p} N}{R_{0}^{2} C}\right)+\frac{K_{p}}{r_{p}}\left(s+\frac{2\left(1-r_{p}\right) N}{R_{0}^{2} C}\right)}{\frac{1}{1-r_{p}}\left(s+\frac{2 r_{p} N}{R_{0}^{2} C}\right)} \cdot C_{r e d}(s) \\
C_{\text {red }}(s)=\frac{L_{\text {red }} \times K}{s+K}, P_{\text {tcp }}(s)=\frac{\frac{R_{0} C^{2}}{2 N^{2}}}{s+\frac{2 N}{R_{0}^{2} C}}, \\
P_{\text {queue }}(s)=\frac{\frac{N}{R_{0}}}{s+\frac{1}{R_{0}}}, L_{\text {red }}=\frac{p_{\text {max }}}{\max _{\text {th }}-\min _{\text {th }}}, K=-\frac{\log _{e}(1-\alpha)}{\delta}
\end{gathered}
$$

Thus, assume the delay of the network is a constant $\mathrm{R}$, the input time delay system of TCP/AQM network can be presented using the following equations:

$$
\begin{aligned}
\dot{X}(t) & =\left[\begin{array}{cc}
-\frac{2\left(1-r_{p}\right) N}{R^{2} C} & 0 \\
\frac{\left(1-r_{p}\right) N}{R} & -\frac{1}{R}
\end{array}\right]\left[\begin{array}{c}
\delta W(t) \\
\delta q(t)
\end{array}\right]+\left[\begin{array}{c}
-\frac{R C^{2}}{2 N^{2}\left(1-r_{p}\right)^{2}} \\
0
\end{array}\right] \delta p(t-R) \\
& =A X(t)+B u(t-R) \\
Y(t) & =\left[\begin{array}{ll}
1 & 0 \\
0 & 1
\end{array}\right]\left[\begin{array}{c}
\delta W(t) \\
\delta q(t)
\end{array}\right]=C X(t)
\end{aligned}
$$

Thus, we have:

$$
\begin{aligned}
& X(t)=\left[\begin{array}{c}
\delta W(t) \\
\delta q(t)
\end{array}\right], A=\left[\begin{array}{cc}
-\frac{2\left(1-r_{p}\right) N}{R^{2} C} & 0 \\
\frac{\left(1-r_{p}\right) N}{R} & -\frac{1}{R}
\end{array}\right], B=\left[\begin{array}{c}
-\frac{R C^{2}}{2 N^{2}\left(1-r_{p}\right)^{2}} \\
0
\end{array}\right], \\
& C=\left[\begin{array}{ll}
1 & 0 \\
0 & 1
\end{array}\right], u(t-R)=\delta p(t-R)
\end{aligned}
$$

If we set $r_{p}$ to be 0 , then the model designed for PC-PI controller can also be used for a simple PI controller with Kalman predictor (KPI).

In IPv6 network, the router can be informed with all three variables needed, $\delta W(t) \delta p(t)$ and $\delta q(t)$, by IPv6 header to predict the next state of the queue size. Considering the limitation of the IPv6 header capacity, it is not recommended for all of the packets to carry the current window size when they are transmitted. Congestion affects both sides of the network. TCP sources can sense packet droppings via duplicated acknowledgement packets. Routers also detect queue sizes which are larger than normal. We could suggest that TCP sources label their outgoing packets with the current window sizes whenever they sense congestion. But usually when a TCP source is aware of the congestion, it returns to the initial window size, which is really small. In this case, it is not necessary for the TCP source to communicate to the router the size of its next transmission, as its importance is rather weak compared to the overall workload. Additionally the source does not really have global supervision of the entire network. Hence, it is more reasonable for the router to trigger a Global Window-Size-Marking Announcement (GWA) when it senses congestions.

\section{Simulations}

The network topology used in the following simulations is 
shown in Figure 7.

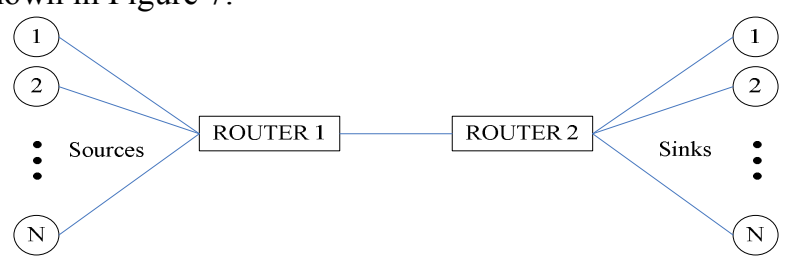

Fig. 7. Network topology

\section{A. PI and PC-PI}

Based on suggestions in [5], we give parameters to the controller as follow:

$$
\begin{aligned}
& N^{-}=60, R^{+}=0.25 \mathrm{~s}, \mathrm{C}=2500 \text { packet } / \mathrm{s}, T=1 / 200 \mathrm{~Hz}=5 \mathrm{~ms} \\
& \omega_{g}=\frac{2 N^{-}}{\left(R^{+}\right)^{2} C}=0.768 \mathrm{rad} / \mathrm{sec} \\
& K_{p i}=\omega_{g}\left|\frac{\left(\frac{j \omega_{g}}{p_{\text {queue }}}+1\right)}{\frac{\left(R^{+} C\right)^{3}}{\left(2 N^{-}\right)^{2}}}\right|=4.613 e-6 \\
& a=\frac{K_{p i}}{\omega_{g}}+\frac{T K_{p i}}{2}=6.016 e-5, b=\frac{K_{p i}}{\omega_{g}}-\frac{T K_{p i}}{2}=5.994 e-5
\end{aligned}
$$

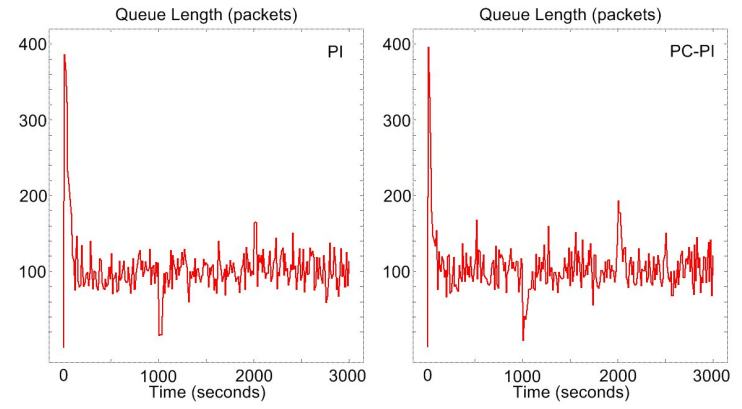

Fig. 8. PI and PC-PI Queue Sizes

Since the minimum number of flow is $\mathrm{N}^{-}=60$ and the maximum value of propagation delay is $\mathrm{R}^{+}=250 \mathrm{~ms}$, we set the network with the time varying dynamics and the mixture of $180 \mathrm{ftp}$ and $100 \mathrm{http}$ flows. The propagation delays are uniformly around $50 \mathrm{~ms}$, with the average packet size being 500 Bytes. During $\mathrm{t}=0 \sim 1000 \mathrm{~s}, 180$ FTP flows are active. At $\mathrm{t}$ $=1000 \mathrm{~s}$, one third of the flows drop out of the network and re-join the network at $\mathrm{t}=2000 \mathrm{~s}$. $q_{\text {ref }}$ for the PI controller was chosen to be 100 packets. Queue Buffer is 400 packets.

In Figure 8, faster response time as well as the regulation of the output to a constant value by both PI and PC-PI control is clearly observed. Both of PI and PC-PI controllers are largely insensitive to the load level variations and attempt to regulate the queue length to the same value of 100 . However, due to the large sized Priority List and the increment of the computation required to maintain it, the oscillation in PC-PI is much larger than under PI controller.

From Figure 9, we can observe the fact that PC-PI has demonstrated its ability of providing higher QoS to High-Priority flows.
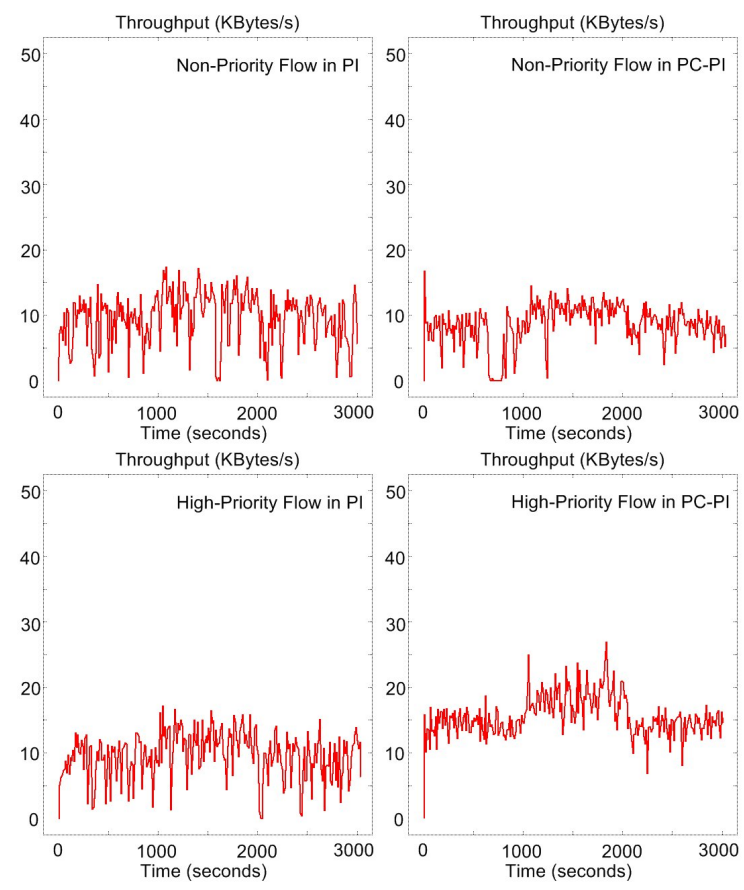

Fig. 9. PI and PC-PI Throughputs

\section{B. $P C-P I$ and DSPCPI}

In this simulation we reduce $\mathrm{N}$ into $20 \mathrm{FTP}$ flows, set target queue size to 50 and buffer size 150 . We use DSPCPI controller to compare the performance with PCPI controller and let half of the dataflow drop off the network at $t=1000$ seconds and rejoin at $\mathrm{t}=2000$ seconds to cause some oscillation of the load level. The queue length plots for the PC-PI and DSPCPI controllers are depicted in Figure 10 and Figure 11. DSPCPI controller responses faster than PCPI controller, and does not have any overshoot.

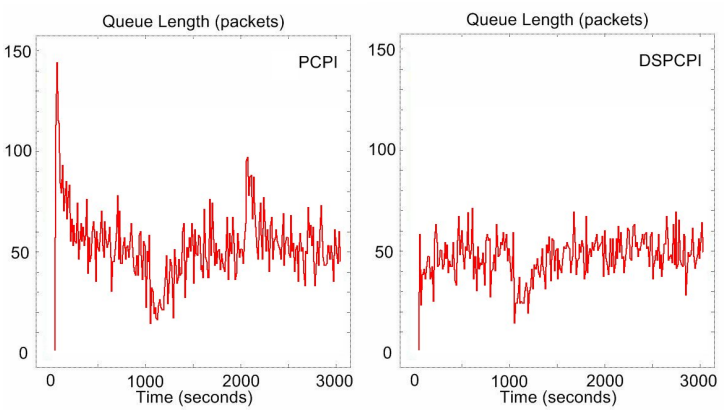

Fig. 10. PC-PI and DSPCPI Queue Sizes

\section{C. $P C$-PI and KPCPI}

Referring to the complete stability region for PI-AQM controllers provided in [10], we first set up a network with 60 FTP flows including 6 High-Priority flows, with $\mathrm{C}=3750$ packets/second, $\mathrm{R}=0.246$ seconds, $\mathrm{q}_{0}=50$ packets. The gains for $\mathrm{P}$ controller and I controllers are $\mathrm{K}_{\mathrm{p}}=\mathrm{e}-4, \mathrm{~K}_{\mathrm{i}}=6 \mathrm{e}-5$. The queue size outputs under PCPI and KPCPI controllers are presented in Figure 12.

We then reduce the link capacity to $\mathrm{C}=1250$ packets/second, $\mathrm{R}=0.22$ seconds, $\mathrm{q} 0=50$ packets. The gains for $\mathrm{P}$ controller and I controllers are $\mathrm{K}_{\mathrm{p}}=7.5546 \mathrm{e}-4, \mathrm{~K}_{\mathrm{i}}=$ 1.4984e-3. The queue size outputs are presented in Figure 13.

Finally, we increase the number of flows to 75 FTP, and 
reduce the propagation delay to 0.15 seconds. $K_{p}$ and $K_{i}$ are set to be 0.0044 and 0.0233 respectively. The queue sizes are presented in Figure 14.
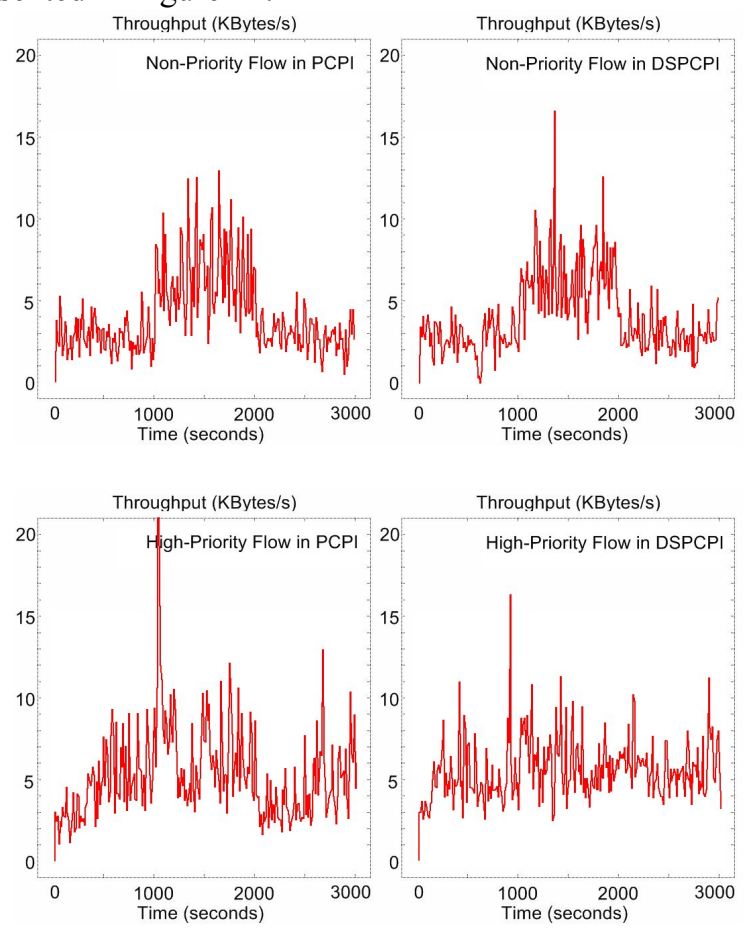

Fig. 11. PC-PI and DSPCPI Throughputs

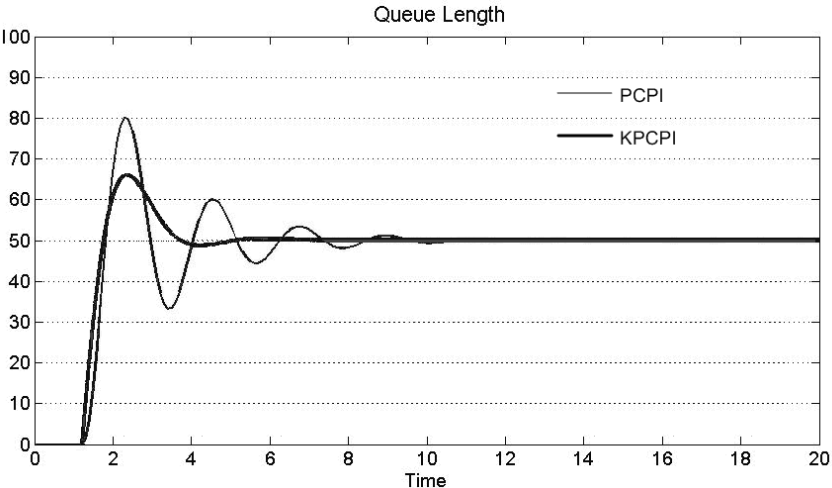

Fig. 12. TCP/AQM with PCPI and KPCPI Controller Queue Length

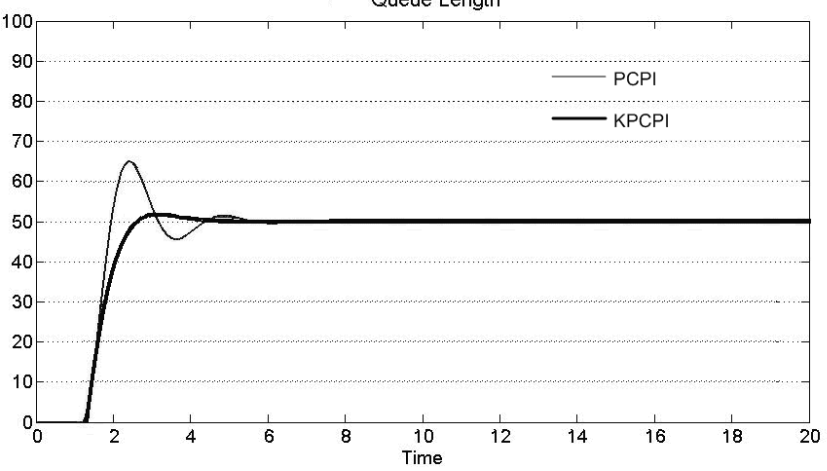

Fig. 13. TCP/AQM with PCPI and KPCPI Controller

\section{CONCLUSIONS}

PI controller is considered to be the natural choice of queue management. When IPv6 brings flow labeling ability to data packets, it is possible for the PI controllers to have more meticulous QoS consideration with the support of Priority Checking Mechanism. In this paper, we have presented PI controllers with Priority Checking Mechanism for TCP/IPv6 networks.

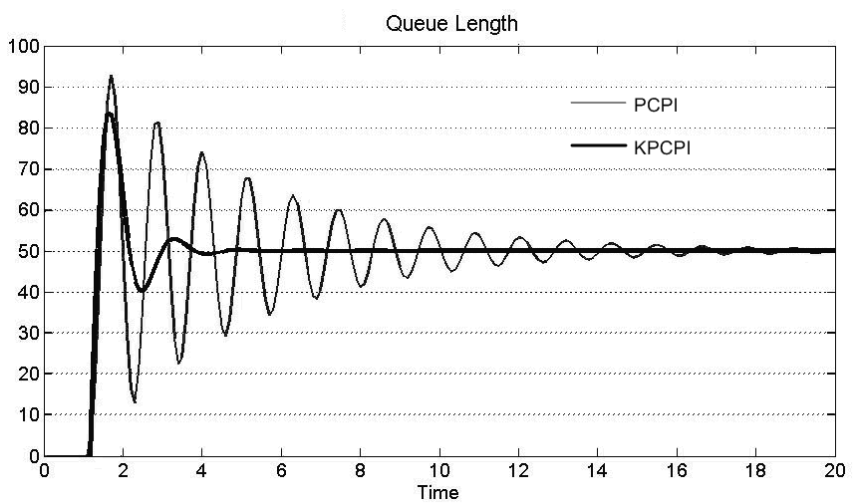

Fig. 14. TCP/AQM with PCPI and KPCPI Controller

To make up the time delay caused by such mechanism, Kalman predictor is used to work in conjunction with PC-PI controllers. Simulation results in both NS2 and SIMULINK have shown remarkable improvement in system performance. Simulations based on more complex network topology, such as multi-domain DiffServ network, are a topic for future study.

\section{ACKNOWLEDGMENT}

Thanks to Fang YAO, Wu CHEN, Khusvinder GILL, Lei LIU and Dr. Jinghua ZHANG for many productive discussions.

\section{REFERENCES}

[1] B. Braden, D. Clark, J. Crowcroft, et al., "Recommendations on queue management and congestion avoidance in the Internet", IETF RFC2309, April 1998.

[2] C. Hollot, V. Misra, D. Towsley, W.B. Gong, "A control theoretic analysis of RED", in: Proc. of IEEE INFOCOM'01, Anchorage, Alaska, 2001, pp. 1510-1519.

[3] Wu CHEN, Yunqiu LI, Shuang-Hua YANG, "An Average Queue Weight Parameterization in a Network Supporting TCP with RED", IEEE ICNSC, 2007, pp. 590-595.

[4] M. May, J. Bolot, C. Diot, B. Lyles, "Reasons not to deploy RED", in: Proc. of IWQoS'9, 1997.

[5] C. Hollot, V. Misra, D. Towsley, and W. Gong, "Analysis and design of controllers for AQM routers supporting TCP flows", IEEE Trans. On Automatic Control, vol. 47, Jun. 2002, pp. 945-959.

[6] WRED: available online at : http://www.cisco.com/univercd/cc/td/doc/product/software/ios112/ios $112 \mathrm{p} / \mathrm{gsr} /$ wred gs.htm

[7] Clark D. and Fang W., "Explicit Allocation of Best Effort Packet Delivery Service", IEEE/ACM Transactions on Networking, V.6 N.4, August 1998.

[8] Li, Y. and Yang, S., "An New RED for the Next Generation Internet IPv6", Proceedings of the PREP conference, Lancaster, UK, 2005, pp 151-152.

[9] C. Hollot, V. Misra, D. Towsley, and W.-B. Gong. On designing improved controllers for AQM routers supporting TCP flows. In Proceedings of IEEE/INFOCOM, April 2001.

[10] Michael S. Branicky, and Stephen M. Phillips,. "Complete Stability Region Characterization. for PI-AQM", SIGBED Review, Vol 3 No. 2, April 2006, pp.1-6. 